\title{
Eine Charta zur Zusammenarbeit - ohne Zusammenarbeit?
}

Jürg Schlup

Präsident der FMH

\begin{abstract}
Zusammenarbeit der Gesundheitsberufe
Die interprofessionelle Zusammenarbeit unter den Gesundheitsberufen wird zweifellos an Bedeutung gewinnen, weil eine gute Gesundheitsversorgung künftig noch mehr Koordination erfordern wird: Das medizinische Wissen wächst beständig, so dass seine Anwendung mit mehr Spezialisierungen einhergeht. Innerhalb dieses sich verbreiternden Feldes medizinischer Kompetenzen entstehen neue Gesundheitsberufe und die bestehenden entwickeln sich weiter. Zudem stellen die zunehmenden Teilzeittätigkeiten oder die Arbeit in Versorgungszentren höhere Anforderungen an die Zusammenarbeit.
\end{abstract}

\section{Sowohl die Erstellung der Charta als auch die einge- räumten Möglichkeiten zu ihrer Modifikation erlaubten keine echte Beteiligung legitimierter Berufsgruppen- vertreter nach demokratischem Verständnis.}

1 Heinen MM. et al. Nurses' intention to leave their profession: A cross sectional observational study in 10 European countries. International Journal of Nursing Studies. 2013;50:174-84

\section{Korrespondenz:}

$\mathrm{FMH}$

Dr. med. Jürg Schlup

Elfenstrasse 18

CH-3000 Bern 15

Tel. 0313591111

Fax 0313591112
Die von der SAMW vorgelegte Charta «Zusammenarbeit der Fachleute im Gesundheitswesen» widmet sich somit einem wichtigen Thema, das zudem von erheblicher Bedeutung für die Qualität der Patientenversorgung und die Arbeitsmotivation der Medizinalund Gesundheitsfachpersonen ist. Die Erfahrungen mit der interprofessionellen Zusammenarbeit im Versorgungsalltag sind sehr positiv. Und auch die Forschung zeigt, dass die Schweiz bspw. bei der Zusammenarbeit zwischen Ärzten und Pflegenden im internationalen Vergleich sehr gut abschneidet [1].

\section{Weiterentwicklung der interprofessionellen} Zusammenarbeit: Anspruch und Wirklichkeit Auch Gutes zu verbessern und weiterzuentwickeln ist richtig.

Die Erarbeitung einer Charta als «Ausdruck für den Geist der Zusammenarbeit der Gesundheitsfachleute» hätte auch die volle Unterstützung der FMH gefunden, wäre sie durch demokratisch legitimierte Vertreter derjenigen Berufsgruppen erfolgt, an die sie sich richtet. Dass diese Charta jedoch durch eine Gruppe erarbeitet wurde, die sich mehrheitlich aus Personen ohne nachvollziehbare Legitimation ihres jeweiligen Berufsstands zusammensetzt, schränkt ihre Bedeutung deutlich ein. Auch dass in dieser Ar- beitsgruppe ohne weitere Begründung einige Gesundheitsberufe durch mehrere Personen, andere durch Einzelpersonen und wiederum andere - wie z. B. Chiropraktoren, Hebammen oder Medizinische Praxisassistentinnen - gar nicht vertreten waren, reduziert den Anspruch der Charta an die hier proklamierte «interprofessionelle Zusammenarbeit» .

Nicht nur die Erstellung der Charta, auch die in der Folge eingeräumten Möglichkeiten zur Modifikation erlaubten keine echte Beteiligung legitimierter Berufsgruppenvertreter nach demokratischem Verständnis: Den ersten Entwurf liess man «ausgewählte Experten» kommentieren. Zur zweiten Fassung führte man eine breite Vernehmlassung durch. Die Tatsache, dass «in Ärztekreisen eine grössere Skepsis spürbar» war und «zahlreiche Institutionen» wünschten, «dass sie stärker in den Ausarbeitungsprozess eingebunden würden», veranlasste die SAMW nicht, entsprechende Anpassungen vorzunehmen.

\section{Weiterentwicklung der interprofessionellen Zusammenarbeit: Legitimation und Transparenz als zwingende Voraussetzungen} Der Erstellungsprozess der Charta zeigt, dass es zweckmässiger gewesen wäre, zunächst die Spielregeln für die gemeinsame Weiterentwicklung der «Zusammenarbeit der Fachleute im Gesundheitswesen» festzulegen. Ein solcher Kodex sollte z. B. den Einbezug der Interessensgruppen sowie die Transparenz von Auswahlprozessen und der Berücksichtigung von Kritik zum Gegenstand haben. Auch das Gewicht, das Experten gegenüber demokratisch legitimierten Berufsgruppenvertretern erhalten dürfen, wäre zu thematisieren.

Die FMH begrüsst, dass die gemeinsame Entwicklung interprofessioneller Leitlinien den Berufs- und Fachverbänden obliegen soll; eine Vorwegnahme der darin wünschenswerten Inhalte durch die Charta lehnen wir jedoch ab.

\section{Zusammenarbeit der Gesundheitsberufe: Und wo liegt die Nahtstelle zum Patienten?} Auch die Patienten als wichtigste und mit Abstand grösste Interessensgruppe im Gesundheitswesen wurden bei der Erstellung der Charta - entgegen ihrer «Kernelemente und Verpflichtungen» - offenbar nicht partnerschaftlich eingebunden. Weder in der Arbeitsgruppe noch im Experten-Board, weder auf der Vernehmlassungs- noch auf der Teilnehmerliste des 
Symposiums zur Charta findet sich ein entsprechender Vertreter.

Der Patient, dessen Behandlung mit der Charta optimiert werden soll, ist sowohl bei der Erarbeitung des Dokuments, als auch in dessen Inhalten nicht berücksichtigt worden. Es bleibt offen, inwiefern ihm für seine Behandlung zukünftig noch ein klar verantwortlicher Ansprechpartner bliebe, würden Kernforderungen der Charta - wie die Aufteilung von Aufgaben und Verantwortung unter den Berufsgruppen «je nach Situation» - umgesetzt. Auch wer ihm gegenüber im Schadensfall bei flexiblen Regelungen und überlappenden Verantwortungsbereichen haftet, ist damit fraglich. Die Rolle des Arztes als verantwortlicher Behandlungspartner, der die Gesundheitsversorgung des Patienten in dessen Sinne koordiniert, wird verworfen, ohne ein neues Gegenüber zu benennen. menlaufen. Dies erhöht die Behandlungsqualität und vermeidet Doppelspurigkeiten.

Die «Beschreibung von Kompetenzen und Verantwortlichkeiten in konkreten Arbeitssituationen» erscheint zudem angesichts der nicht abschliessend aufzählbaren Situationen kaum zu leisten. Situative Entscheidungen über Teilverantwortungen sind unübersichtlich und fragmentieren die Behandlung. Darüber hinaus geht die Aufteilung der Verantwortung gegenüber dem Patienten auch mit einer Rechtsunsicherheit für die beteiligten Medizinal- und Gesundheitsberufe einher, v. a. im ambulanten Bereich. Die Verantwortung läge nicht mehr klar beim Arzt, sondern je nach Situation und Behandlungseinrichtung bei jemand anderem. Bereits ganz normale Behandlungen würden sich bei Verzicht auf einen delegierenden klaren Ansprechpartner verkomplizieren, von Fällen mit Meinungsunterschieden unter den Fach-

\section{Die Rolle des Arztes als verantwortlicher Behandlungspartner, der die Gesundheitsversorgung des Patienten in dessen Sinne koordiniert, wird verworfen, ohne ein neues Gegenüber zu benennen.}

\section{Eine Umverteilung von Verantwortung zulasten aller Beteiligter?}

Die eben angesprochenen Ausführungen zur Verantwortung, die «entsprechend den fachlichen Kompetenzen wahrgenommen» werden soll, bilden das inhaltliche Kernproblem der Charta. Der zu wenig differenzierte und juristisch nicht eindeutige Umgang mit dem Begriff «Verantwortung» lässt verschiedene Interpretationen des Inhalts der Charta zu. Als Stossrichtung lässt sich klar erkennen, dass es um die «Übernahme zusätzlicher Verantwortung» durch einzelne Gesundheitsberufe geht, konkretisiert werden in diesem Zusammenhang «erweiterte[n] Diagnose-, Verordnungs- und Abrechnungskompetenzen». Die Orientierung «an hierarchischen Kriterien und/oder traditionellen Berufsbildern» wird zugunsten eines situativen Führungsverständnisses abgelehnt. Für «konkrete Arbeitssituationen [sollen] in einem gemeinsamen Prozess» Richtlinien erarbeitet werden, die teilweise organisationsübergreifend, teilweise institutionsspezifisch Gültigkeit haben.

Ausgeblendet wird hierbei unter anderem, dass Ärzte bereits heute tagtäglich bereit sind, «je nach Situation Aufgaben abzugeben», und dies im Rahmen des bewährten Delegationsmodells tun. Dieses basiert auf der ärztlichen Kompetenz und hat den Vorteil, dass die Verantwortung für alles, was getan oder unterlassen wird, jederzeit eindeutig zugeordnet werden kann. Weiterhin hat dieses Modell angesichts zunehmender Spezialisierung und einer grösser werdenden Zahl von Gesundheitsberufen den Vorteil, dass die Fäden der Behandlung eines Patienten an einer zentralen und hinreichend kompetenten Stelle zusam- personen oder gar Schadensfällen und Rechtsstreitigkeiten ganz zu schweigen.

\section{Die Charta - ein dynamisches Dokument?}

Angesichts des oben beschriebenen Entstehungsprozesses der Charta, in dem ein Einwirken auf die Inhalte von Seiten der Ärzteorganisationen kaum möglich war, erscheint die Ankündigung, die Charta sei ein dynamisches Dokument, wenig glaubwürdig. Die FMH versteht unter aktiver Mitwirkung an einem «Entwicklungs- und Veränderungsprozess» nicht, dass sie gelegentlich zum Erarbeiteten Stellung nehmen darf und anschliessend abwarten muss, ob einzelne Kritikpunkte ggf. zu leichten Anpassungen führen oder auch nicht.

Zudem sieht die Charta - entgegen ihrem angeblich vorläufigen bzw. dynamischen Charakter - bereits eine sehr konkrete Umsetzung ihrer Inhalte vor. Statt ihrer Weiterentwicklung eine gemeinsame Überarbeitung mit demokratisch legitimierten Vertretern voranzustellen, sind vielmehr bereits ihre Verwendung als «Lehrmittel» und als Basis für den «institutionalisierten Kontakt der Verbände» vorgesehen, sowie ein Monitoring und «gegebenenfalls» Anpassungen nach zwei Jahren geplant. Auch die Umsetzung in Form eines gemeinsamen Einsatzes für Gesetzes-, Verordnungs- und Tarifänderungen verkennt, dass dem eine Konsensfindung über zentrale Inhalte vorangehen müsste. Angesichts ihrer Entstehungsgeschichte sowie in Anbetracht ihrer strittigen Inhalte wird die Charta diese sich selbst zugeschriebenen Rollen kaum wahrnehmen können. 